\title{
ON THE DETERMINANT OF AN AUTOMORPH OF A NONSINGULAR SKEW-SYMMETRIC MATRIX
}

\author{
JOHN WILLIAMSON
}

Let $G$ be the skew-symmetric matrix of order $2 n$,

$$
G=\left(\begin{array}{cc}
0 & E_{n} \\
-E_{n} & 0
\end{array}\right)
$$

where $E_{n}$ is the unit matrix of order $n$. If $F$ is a matrix which satisfies

$$
F G F^{\prime}=G,
$$

then $|F|^{2}=1$, so that $|F|= \pm 1$. That $|F|=+1$ is well known and is in fact a consequence of a theorem of Frobenius.* A simple proof communicated to me by Professor Wintner depends on the polar factorization of $F$, which reduces the problem at once to the case in which $F$ is orthogonal. This proof is, of course, not valid in any field. It is our intention here to give a simple direct proof, applicable in any field, of the fact that $|F|=+1$.

On writing $F$ as a matrix of matrices of order $n, F=\left(F_{i j}\right),(i, j=1,2)$, we have, as a consequence of (1),

$$
\begin{aligned}
& F_{11} F_{12}^{\prime}-F_{12} F_{11}^{\prime}=F_{21} F_{22}^{\prime}-F_{22} F_{21}^{\prime}=0, \\
& F_{11} F_{22}^{\prime}-F_{12} F_{21}^{\prime}=F_{22} F_{11}^{\prime}-F_{21} F_{12}^{\prime}=E_{n} .
\end{aligned}
$$

Let $\left|F_{11}\right| \neq 0$. Then

$$
F=\left(\begin{array}{ll}
F_{11} & F_{12} F_{11}^{\prime} \\
F_{21} & F_{22} F_{11}^{\prime}
\end{array}\right)\left(\begin{array}{cc}
E_{n} & 0 \\
0 & \left(F_{11}^{\prime}\right)^{-1}
\end{array}\right) .
$$

On, applying (2), we have

$$
\begin{aligned}
\left|F_{11}^{\prime}\right||F| & =\left|\begin{array}{ll}
F_{11} & F_{12} F_{11}^{\prime} \\
F_{21} & F_{22} F_{11}^{\prime}
\end{array}\right|=\left|\begin{array}{ll}
F_{11} & F_{12} F_{11}^{\prime}-F_{11} F_{12}^{\prime} \\
F_{12} & F_{22} F_{11}^{\prime}-F_{21} F_{12}^{\prime}
\end{array}\right| \\
& =\left|\begin{array}{ll}
F_{11} & 0 \\
F_{21} & E_{n}
\end{array}\right|=\left|F_{11}\right| .
\end{aligned}
$$

* G. Frobenius, Ueber die schiefe Invariante einer bilinearen oder quadratischen Form, Journal für die reine und angewandte Mathematik, vol. 86 (1879), pp. 44-71; in particular, p. 48. See A. Wintner, On linear conservative dynamical systems, Annali di Matematica Pura ed Applicata, (4), vol. 13 (1934-1935), pp. 105-112. 
Therefore $|F|=+1$, and we have proved the following lemma:

Lemma 1. If $F_{11}$ is nonsingular, $|F|=+1$.

It also follows from (2) that if $F_{12}=F_{21}=0, F_{11} F_{22}^{\prime}=E_{n}$, so that $F_{11}$ is nonsingular and accordingly $|F|=+1$.

Let $P_{i j}$ be the permutation matrix of order $n$, which by post-multiplication interchanges the $i$ th and the $j$ th columns of a matrix, and let $P$ be the diagonal block matrix

$$
P=\left[P_{i j}, P_{i j}\right]=\left(\begin{array}{cc}
P_{i j} & 0 \\
0 & P_{i j}
\end{array}\right) \text {. }
$$

Since $P_{i j}$ is symmetric and involutory, $P G P^{\prime}=G$, the matrix $F P$ satisfies (1), and $|F P|=|F|$. Consequently we have the following lemma:

Lemma 2. Any matrix $F_{1}$ obtained from $F$ by a permutation of its first $n$ columns and the same permutation of its last $n$ columns also satisfies (1) and $\left|F_{1}\right|=|F|$.

The matrix $W=\left(W_{i j}\right),(i, j=1,2)$, where $W_{11}=W_{22}=\left[0, E_{n-1}\right]$ and $W_{12}=-W_{21}=\left[E_{1}, 0\right]$, satisfies (1) and has determinant unity. The matrix $F W$ is obtained from $F$ by replacing the first column by minus the $(n+1)$ st column and the $(n+1)$ st by the first. If, for convenience, we now write $F_{11}=A$ and $F_{12}=B$ and denote the columns of $A$ and $B$ by $a_{i}$ and $b_{i}$, respectively, we have, as a consequence of Lemma 2 , the following lemma:

Lemma 3. The matrix $A=F_{11}$ in $F$ may be replaced by $C=\left(c_{1}, c_{2}, \cdots\right.$, $\left.c_{n}\right)$, where $c_{i}=a_{i}$ or $-b_{i}$.

Therefore by Lemma 1 , since $|W|=+1$, we have our fourth lemma:

Lemma 4. If there exists a matrix $C$ such that $|C| \neq 0$, then $|F|=+1$.

Let every determinant of order $n$ formed from $(A, B)$, in which less than $r$ pairs of columns $a_{i}, b_{i}$ occur with the same suffix $i$, be zero, but let at least one determinant with exactly $r$ pairs of columns $a_{i}, b_{i}$ be different from zero. As a consequence of Lemma 2 there is no loss in generality in assuming that

$$
\left|a_{1} b_{1} X\right| \neq 0,
$$

where the matrix $X$ contains exactly $r-1$ pairs of columns $a_{i}, b_{i}$ with the same subscript $i$ and does not contain either of the columns $a_{2}$ or $b_{2}$. Let $Q$ be the diagonal block matrix 


$$
Q=\left[\left(\begin{array}{ll}
1 & 1 \\
0 & 1
\end{array}\right), E_{n-1},\left(\begin{array}{rr}
1 & 0 \\
-1 & 1
\end{array}\right), E_{n-2}\right]
$$

Then $|Q|=+1, Q G Q^{\prime}=G$, and $F Q$ satisfies (1). The matrix of the first $n$ rows of $F Q$ is $(H, K)$, where the $n$ columns $h_{j}$ of $H$ are given by

$$
h_{j}=a_{j}, \quad j \neq 2, \quad h_{2}=a_{1}+a_{2},
$$

and the $n$ columns $k_{i}$ of $K$ by

$$
k_{i}=b_{i}, \quad i \neq 1, \quad k_{1}=b_{1}-b_{2} .
$$

Since the matrix $X$ in (3) does not contain any of the columns $a_{1}, b_{1}$, $a_{2}, b_{2}$, it follows from (4) and (5) that the matrix $T=\left(h_{2} k_{1} X\right)$ is a submatrix of $(H, K)$, which contains exactly $r-1$ pairs of columns $h_{i}, k_{i}$ with the same suffix $i$, and that

$$
\begin{aligned}
|T| & =\left|a_{1}+a_{2}, b_{1}-b_{2}, X\right| \\
& =\left|a_{1} b_{1} X\right|-\left|a_{1} b_{2} X\right|+\left|a_{2} b_{1} X\right|-\left|a_{2} b_{2} X\right| .
\end{aligned}
$$

But, by hypothesis,

$$
\left|a_{1} b_{2} X\right|=\left|a_{2} b_{1} X\right|=0 \text {. }
$$

Since $\left|a_{1} a_{2} X\right|$ is also zero and $\left|a_{1} b_{1} X\right|$ is not zero by (3), $a_{2}$ and $b_{2}$ are both linear combinations of the $n-1$ columns of the matrix $\left(a_{1} X\right)$. Hence $\left|a_{2} b_{2} X\right|=0$ and, as a consequence of (6) and (7), $|T|=\left|a_{1} b_{1} X\right|$ $\neq 0$.

Therefore in $(H, K)$ there is one nonzero subdeterminant of order $n$ which contains exactly $r-1$ pairs of columns $h_{i}, k_{i}$ with the same suffix $i$.

Now $|F Q|=|F|$, and $F Q$ satisfies (1). Further, the matrix $C$ in Lemma 4 contains exactly $r=0$ pairs of columns $a_{i}, b_{i}$. By a simple induction proof we therefore have the following lemma:

LEMma 5. If in $(A, B)$ there is one nonzero subdeterminant of order $n$, which contains $r \leqq n$ pairs of columns $a_{i}, b_{i}$ with the same suffix $i$, then $|F|=+1$.

Since any matrix $F$ which satisfies (1) is nonsingular, the rank of $(A, B)$ is $n$ and Lemma 5 implies the following statement:

THEOREM. If $F G F^{\prime}=G,|F|=+1$.

This proof is valid in any field.

The John Hopkins University 